\title{
Cast Iron Components with Intelligence
}

\author{
Lennart Elmquist ${ }^{1, a^{*}}$, Raul Carlsson ${ }^{1, b}$ and Christer Johansson ${ }^{2, \mathrm{c}}$ \\ ${ }^{1}$ Swerea SWECAST, P.O. Box 2033, SE-550 02 Jönköping, Sweden \\ ${ }^{2}$ RISE Acreo AB, P.O. Box 53071, SE-400 14 Göteborg , Sweden \\ alennart.elmquist@swerea.se, ${ }^{b}$ raul.carlsson@swerea.se, ${ }^{c}$ christer.johansson@ri.se
}

\begin{abstract}
Keywords: cast iron, integrated sensors, digitalized castings, interfacial phenomena, intelligent castings, intelligent cast components
\end{abstract}

\begin{abstract}
The paper describes a project with the aim to develop communicating and functional cast iron components in smart systems. The concept is based on sensors integrated into cast iron components; this will influence not only the component but also the casting process. Among the technical challenges is how to choose a sensor solution that cost-efficiently and with minimal environmental impact can be integrated into the component during the casting process, and especially without being damaged during mold filling and the high pouring temperature. Another challenge is how the iron will interact and interfere with sensor signals and whether an insulating intermediate material is needed or not. Integrating the sensors into the casting makes sensors a natural part of the component, which in turn can lead to more resource efficient designs, increased value added for the casting sector, and a general access to different possibilities of digitalization. The integrated sensors can be used for effective control and monitoring of components when in service and give information about for example how the component is used and what conditions it is exposed to. In other words, the component can tell when maintenance is needed or in worst cases, indicate that something is wrong before a failure will happen. Important measurands can e.g. be elongation, shear, temperature and vibration. Different combinations of sensor materials and insulating materials and their interaction with the cast iron have been investigated. It is shown how the interaction at the interface affects the microstructure and consequently the properties of the cast iron. In the case of insulating materials it is e.g. shown how air gaps are formed and in the case of sensor materials it is shown how a diffusion zone is formed and how this zone depends on the sensor material. How this diffusion zone affects the microstructure is discussed.
\end{abstract}

\section{Introduction}

This work is part of the ongoing development of a new technology that can reduce costs for a wider integration of robust sensors into cast components, and the products in which they are used. This has the potential to revolutionize the digitalization of cast components. Digitalization is the new market potential and consequently of paramount importance to all aspects of industrial production, products, business and markets, and therefore also to the casting industry. Digitalization of cast components means that the evolution of Internet into Internet of Things (IoT) takes the next step towards Internet of Materials (IoM), Fig 1. This paper presents current research and the initial efforts to evolve also cast iron and cast iron production in that direction.

Today, the casting industry produces mechanically active but electronically passive components, with high requirements on properties and quality for use in a wide range of applications. Electronically active cast components will open for entirely new markets and thus a wider spectrum of applications for embedded robust and cost-efficient sensor systems. The potential for digitalization is the possibility to more efficiently control and monitor different components in applications hard to reach and/or at remote locations in order to reduce for example energy losses or avoid failures by stopping machinery before the failure occurs. Sensors together with cost-efficient sensor functionality can also be used to register user behavior and the actual properties needed during operation. Such valuable data and feedback to the designer can be used advantageously during the design of the next generation of the product. This will for example avoid unnecessary 
oversizing and instead design the component with weight and dimensions optimized for the specific application. Transforming these electronically passive cast iron components into components with intelligence have a huge potential to significantly accelerate the digitalization of all applications where cast iron today are used as mechanically active components.

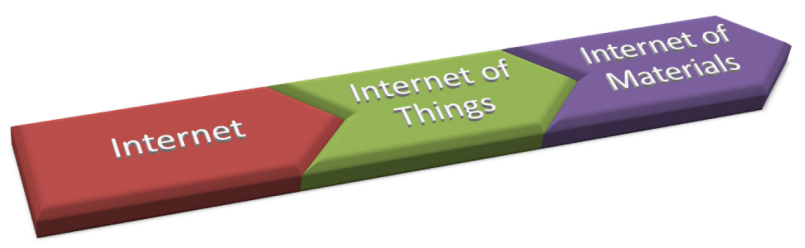

Figure 1. Internet of Materials, IoM [1].

One example of a possible application is sensors in bearings, inside for example windmills at remotely placed sea-based wind farms or at other places difficult to reach. By robustly and continuously monitoring the functioning of the bearings, breakdowns can be avoided and service intervals reduced, and instead maintenance is done when really needed. This also means that the performance of the bearings can be monitored and optimized.

Beyond the immediate technical benefits, embedded sensors also leads to increased value added to the casting industry; through a more resource efficient cast component design and general access for cast components in any application to all possibilities of digitalization. Introducing sensors during casting will increase the market for the foundries and open up for new possibilities. Besides, castings are used in a wide range of applications and the concept with embedded sensors therefore has the potential to add value far outside the casting industry. Embedded sensors may also enable a wider market for digitally controlled and monitored products. Apart from the practical aspects such as robustness and cost-efficiency, the potentials of embedding sensors into cast metal components can also be expected to result in lower weight, smaller component size, and open up for a wider range of design alternatives. This in turn will also open for the possibility to add more sensors in each component, identify new physical locations to sense at, and add new properties to sense.

Today some cast metal components are supplied with surface mounted sensors. These are glued, drilled or screwed onto the already cast component. The additional manufacturing steps needed to do that means extra costs that can be considered as the major threshold for digitalization of the casting industry. Surface mounted sensors also introduce weaknesses during the product life. For example, tensions, vibrations and varying temperatures may lead to decreased robustness and increase the product life cycle cost. Some of these problems may be solved if the sensors are integrated into the component already during casting. Besides, surface mounted sensors means that the increased value added to the component not necessarily will favor the casting industry. Another alternative would be to measure physical properties of the component to monitor changes. However, the physical properties can be measured by the sensors and this is advantageous especially if the component is hard to access.

Figure 2 presents the generic approach used in this work; a wire of deviating material acting as a sensor embedded in the cast metal. Changes in the cast metal will also affect the wire. Changes in the signal through the wire will reflect changes in the cast metal. The wire may be a metal or any other material that can carry a signal. If necessary, the wire will also have to be encapsulated in an insulating material.

Integrating sensors into the component already during casting is not easy and some of the challenges were identified in a pre-study [1]. These challenges include metallurgical and mechanical issues but are also related to the choice of sensor method and sensor signal and the subsequent signal analysis. Mechanical challenges include for example how the embedded sensors affect the material properties. Other challenges involve data science and commercial aspects. In this paper focus is on the metallurgical challenges when integrating sensors in cast iron. 

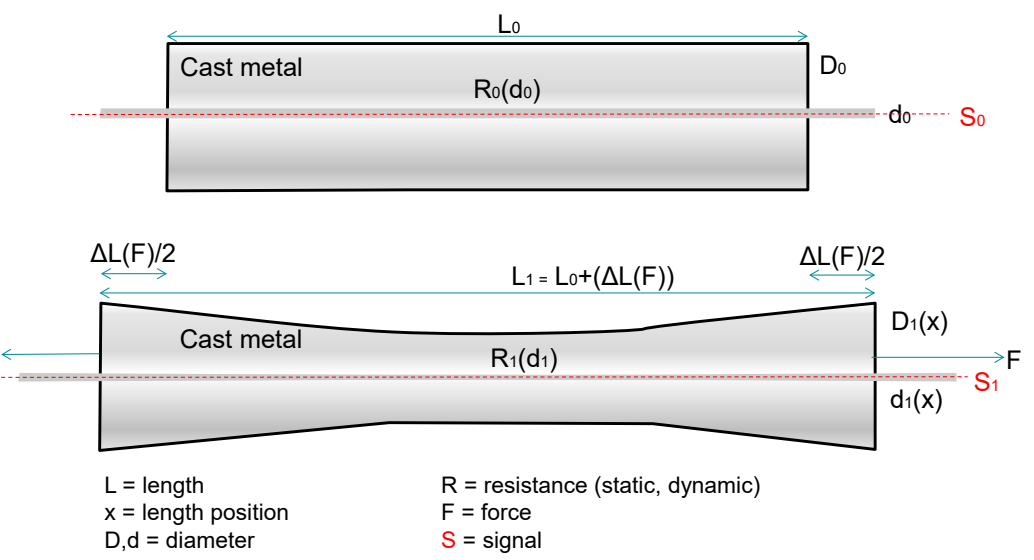

Figure 2. The generic approach used in this work [1].

Metallurgical challenges include for example:

- Volume changes during solidification, which may adversely impact the sensor.

- Material defects impacting mechanical strength and metal properties at micro-level.

- Defects in the surface between cast material and sensor or isolator, such as gas bubbles.

- Intermetallic phases, carbides, brittle structures.

- Diffusion and/or reactions between cast material and sensor or isolator.

- Unwanted solidification structures, such as degenerated graphite.

Metals inevitably shrink during solidification and the subsequent cooling to room temperature. This will affect the interface between the cast material and sensor system due to differences in properties such as thermal expansion. A sensor, with or without insulating material, will create a discontinuity in the cast metal, and as such be considered as a defect in the structure. Casting defects is already a problem, with issues such as porosities and gases formed due to for example chemical reactions and a reduced solubility as the temperature decreases. With the introduction of sensors this complexity will increase. Other challenges are related to the formation of unwanted solidification structures such as intermetallic phases and carbides, constituents in the microstructure that are known to adversely decrease and deteriorate the expected properties. A sensor inside a mold will in practice represent a surface which will influence the solidification structure of the cast metal.

In this paper focus is on one of the challenges, the interface between cast iron and a sensor wire or insulator material. The interface formed and the interaction between the cast iron and any sensor/insulator material is a critical area for stable and reliable properties and is thus one of the key questions. In the case of cast iron all metallurgical challenges described can be expected to occur and besides them also the high temperatures and turbulences during mold filling will challenge the possibility for both positioning and survival of the sensors.

Integrating sensors into cast iron components is quite unusual, or at least belongs to an unpublished research area. There is some research activities related to development of integrating sensors with materials and some of this was presented at the International Conference on SystemIntegrated Intelligence [2]. It is also some of the core activities of The European Technology Platform on Smart Systems Integration - EpoSS [3]. Especially a state of the art with visionary outlook presented by Lehmus at the EpoSS in 2013 [4]. However, apart from this there is not much published about integrating sensors into metals. Especially when it comes to ferrous metals nothing similar can be found. Nearest and most related is the well-developed technique marketed by Fraunhofer IFAM, in which piezo-electric sensors and RFID antennas been integrated into aluminum [5]. This is the most similar approach. Somewhat related to the present work is research done on integrating sensor materials into metals during additive manufacturing [6,7]. Another example is a patent that describes a technique to mount optical fibers into metal [8]. In the present project the starting point is the possibilities and challenges of integrating sensors into the cast metal itself and its influence on the casting process. In other words, in this work the cast component, 
material and process is in focus and the long-term strategic goal is to develop and increase the market for the foundry industry.

The integration is not limited to integrating sensors, but to transform cast metal into also being active sensors by developing the cast material and the casting process. That would eliminate the need for adding unwanted materials and technologies to the cast component.

\section{Experimental Method}

In this work, the interaction between cast iron and different sensor/insulator materials were investigated in a first simple test. Two different cast irons were used; lamellar (LGI) and nodular (SGI) iron. The interaction with six different sensor/insulator materials were investigated, see Table 1. The test geometry used was ordinary cups for thermal analysis, see Fig. 3a. The thermocouple and quartz tube was removed and replaced by the sensor/insulator material to be tested before being filled with the cast material. For Sample no 1, quartz tube, nothing was removed from the cup and the ordinary quartz tube was used as test material. The chemical compositions of the cast materials are shown in Table 2. The nodular iron came from two different treatments but from the same base iron. The LGI samples are marked as B1-B6 and the SGI samples C1-C6.

After cooling the cups were sectioned and the cross-sections investigated using Light Optical Microscopy (LOM). Sample preparation was done according standard procedure. The samples were etched with $2 \%$ Nital to reveal the matrix structure.

Table 1. Sensor/insulator materials tested and their thicknesses.

\begin{tabular}{|l|l|l|}
\hline Sample no. & \multicolumn{1}{|c|}{ Material } & \multicolumn{1}{c|}{ Thickness [mm] } \\
\hline 1 & Quartz tube & 2.5 \\
\hline 2 & $\mathrm{Al}_{2} \mathrm{O}_{3}$ tube & 2.5 \\
\hline 3 & Titanium wire & 0.5 \\
\hline 4 & Tungsten wire & 0.7 \\
\hline 5 & 316L Stainless steel wire, & 0.16 \\
\hline 6 & Kanthal wire (FeCrAl-alloy) & $0.8 \times 0.1$ flat ribbon \\
\hline
\end{tabular}

The first cut was simply done at the center of the sample geometry, producing two separate halves, see Fig 3b. After documentation of the cross-section, one of the halves was further sectioned to smaller samples. The region investigated is shown in Fig 3c.

In some cases the sensor wires could not be found on the cross-section at Cut 1 . In those cases the samples were etched to look for deviations in the graphite and matrix. However, no wires were found. The next step was to make a second cross-section closer to the surface, Cut 2 in Fig $3 \mathrm{~b}$.

Table 2. Chemical compositions of the irons.

\begin{tabular}{llllllllllll}
\hline \multicolumn{1}{c}{ wt \% } & C & Si & Mn & P & S & Cr & Ni & Cu & Sn & Mg & CE \\
\hline LGI & 3.25 & 2.13 & 0.57 & 0.208 & 0.020 & 0.063 & 0.014 & 0.331 & 0.004 & - & 3.88 \\
SGI, ladle 1 & 3.56 & 2.06 & 0.365 & 0.013 & 0.006 & 0.021 & 0.025 & 0.245 & 0.007 & 0.031 & 4.08 \\
SGI, ladle 2 & 3.53 & 2.25 & 0.408 & 0.014 & 0.006 & 0.022 & 0.028 & 0.244 & 0.007 & 0.038 & 4.10 \\
\hline
\end{tabular}

The interfaces were qualitatively investigated to relate the result to the metallurgical challenges earlier identified. The occurrence of defects was investigated, and so also anomalies in the graphite morphology and the matrix structure. 
a)

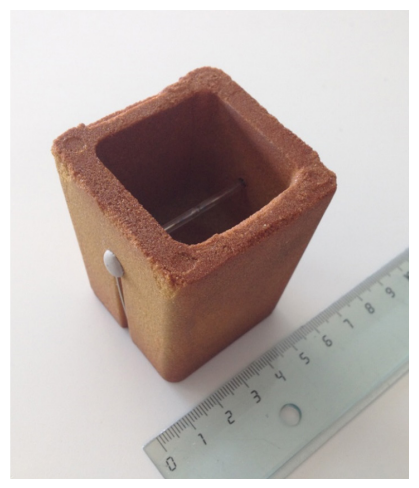

b)

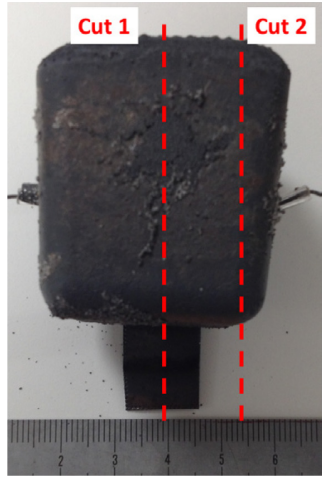

c)

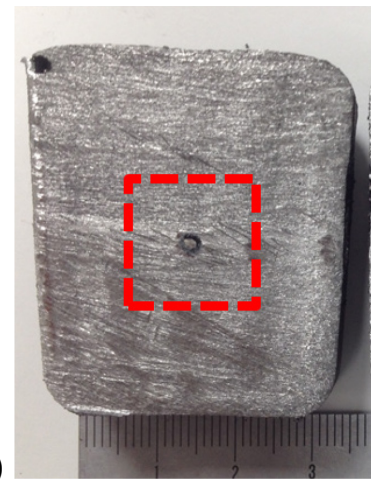

Figure 3. a) Ordinary cups for thermal analysis were used as molds, b) two different cross-sections were investigated and, c) the investigated region was approximately $15 \times 15 \mathrm{~mm}$.

\section{Results and Discussion}

The main focus in this work was to investigate the interaction between cast iron and different sensor/insulator materials, and not the sensor functionality itself. Figure 4 shows three of the final as-cast samples with their sensor/insulator materials.
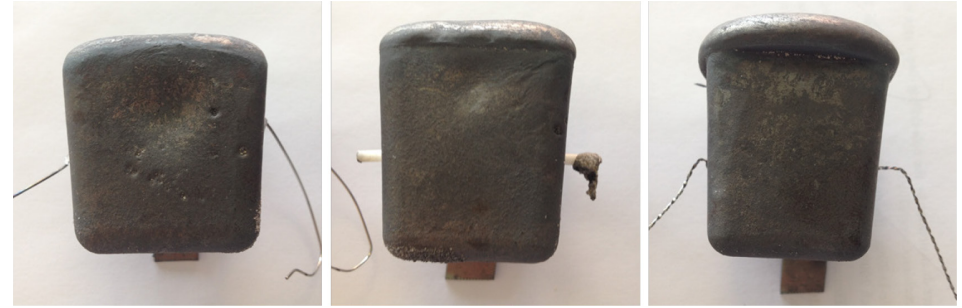

Figure 4. Three of the final as-cast samples (C1, C2 and C6).

The first observation is that all SGI samples have defects to different extents, see Fig 5 . These are of various kinds; some are related to shrinkage while others are gas defects. However, these defects are not necessarily formed due to the sensor/insulator material. Due to these cavities it was difficult to find all sensor/insulator materials on the cross-section. In some cases the materials that should have been located inside the cavity can be expected to have been detached during the cutting. It is also interesting to notice that defects were found on $\mathrm{C} 1$ which actually is an ordinary cup for thermal analysis. The question one can ask is how that influences the result during thermal analysis. Another interesting observation is the depressions found on the surfaces, see Fig. 4. These are present on the samples also having interior defects.

Surprisingly, in the case of lamellar iron there were no defects observed and thus easier to find the sensor/insulator materials, Fig 6. It must however not be forgotten that the investigation on this stage only is done on the center cross-section. This means that minor defects still can be present. Another problem was that some of the wires were not located in the center where expected, and could not even be found on the cross-section examined. In these cases the second cut closer to the surface was made, where the sensor/insulator material was fixed and therefore should be found. The only sensor wire still not found when examining this second cross-section was the stainless steel wire. However, the wire is observed on the outside of B5 so the question is whether it has been dissolved. In the other cases the most probable explanation is that the wires has been attached too loose, and therefore located outside the region investigated on the first cross-section. Once again, it is interesting to notice the differences in outer sunks, or depressions. In general, there is much more tendency for depressions on the SGI samples. 

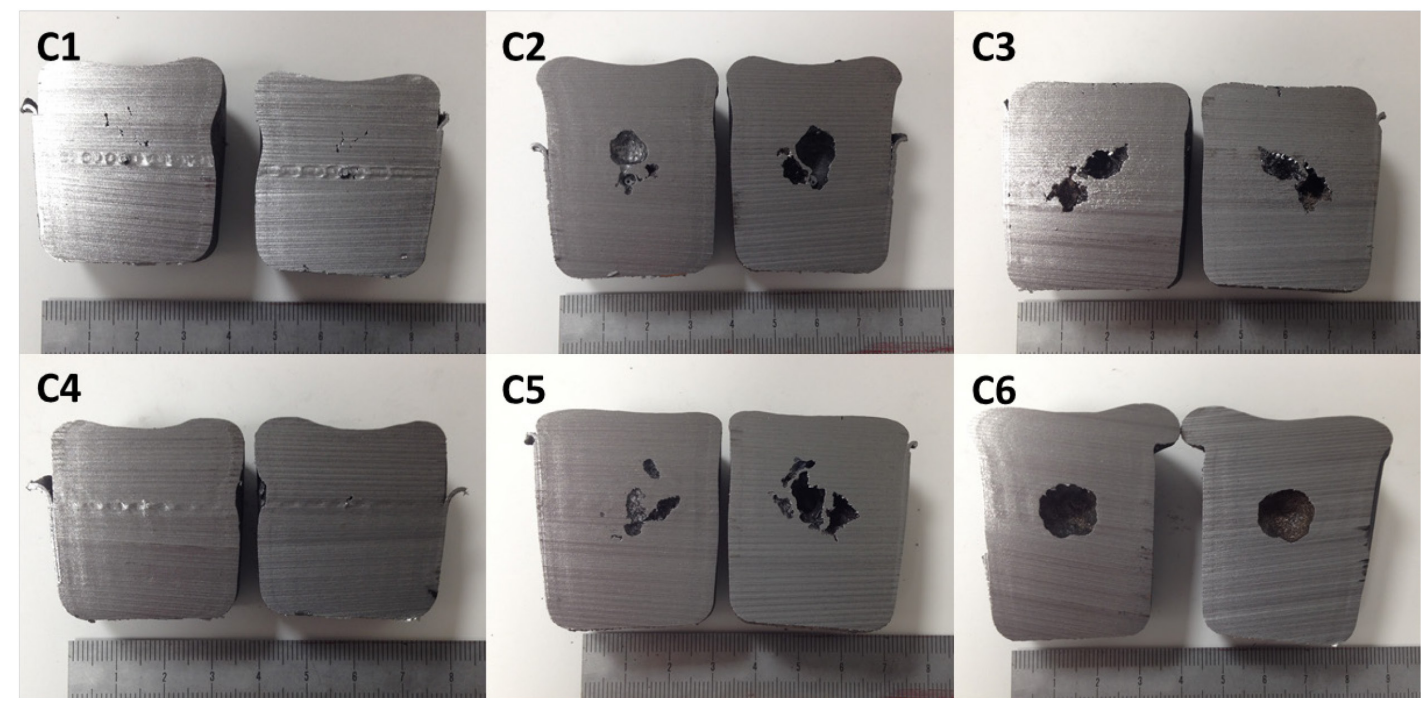

Figure 5. Cross-sections of the SGI samples.
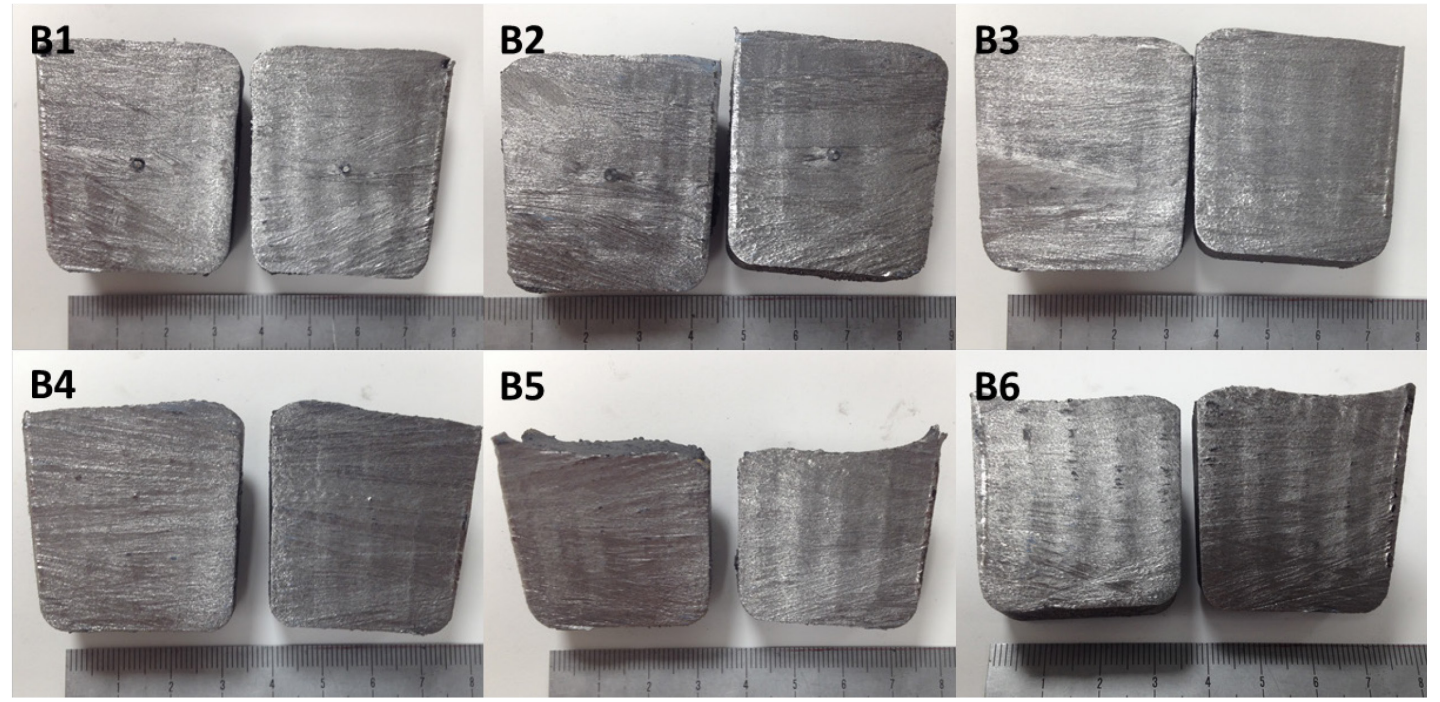

Figure 6. Cross-sections of the LGI samples.

The next step was to investigate the microstructure and interface between the iron and the sensor/insulator material. The first observation is that the sensor/insulator material is not found on any of the samples B3, B5, B6, C3, C5, and C6. In other words, all samples containing titanium, stainless steel, and Kanthal wires. These samples were etched to examine if there were any deviations in graphite morphology or matrix structure that could be used to identify the wires, but nothing was found. Therefore it was decided to instead use the second cut.

Some interesting observations in the microstructure have been summarized in Fig. 7. On sample B1 some minor cracks were found growing from the quartz tube into the iron until they reached a graphite flake. These cracks have also been seen away from the tube. These cracks can also be a result of the cutting. There is some undercooled graphite in the structure but that can of course be a consequence of no inoculation. Close to the tube A-type graphite is formed. In Sample $\mathrm{B} 2, \mathrm{Al}_{2} \mathrm{O}_{3}$ tube, cracks has formed in the alumina that filled the ordinary holes with iron. Graphite is found in the structure and therefore it can be concluded that these cracks were formed before the cutting. On all samples with insulating material a small air gap is found at the interface. In both B3 and C3 carbides are found. Sample C3 also has interfacial problems with large gaps between the wire and the iron. Another observation is that the wire in C3 still is circular but in B3 it has changed shape. Besides, in C3 it is very clear that degenerated graphite has formed. 

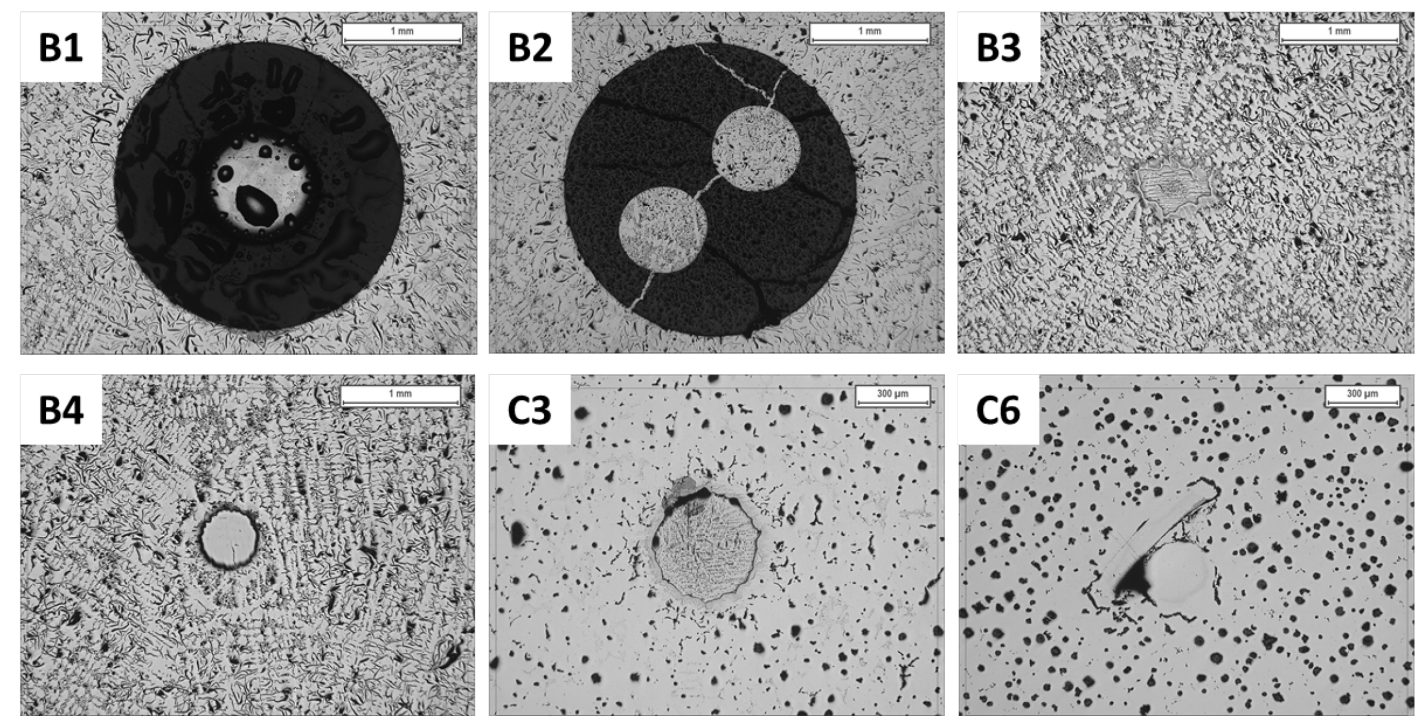

Figure 7. Interaction between iron and sensor/insulator materials for some of the samples.

One strange observation on sample B4 is that the wire should be $0.7 \mathrm{~mm}$ in diameter but on the cross-section it is only about $0.5 \mathrm{~mm}$. This wire also has a crack, see Fig. 8a, and both carbides and an intermediate layer of deviating structure has formed between the wire and the iron, see Fig $8 \mathrm{~b}$. There are different carbides and inclusions formed; some of them have a very characteristic sharp appearance. Other inclusions are distributed not only around the wire and have a characteristic gold/copper color. The strange shape of the wire in C6 depends on the wire that is a twisted flat ribbon and clearly not a good solution for complete bonding with the iron as voids very easily are formed. The matrix structures in both LGI and SGI were mostly pearlitic with some ferrite.

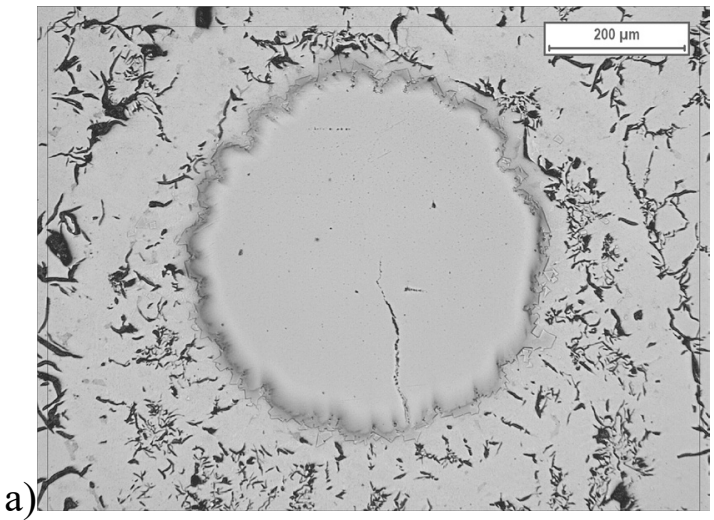

b)

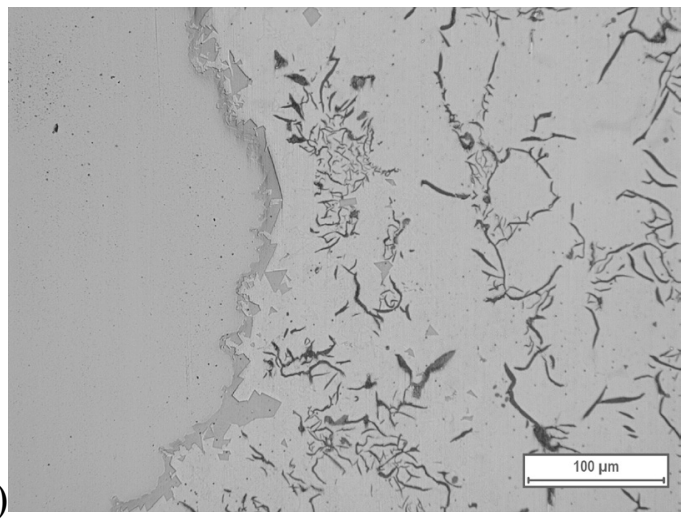

Figure 8. a) Close up of sample B4 reveals both a crack in the sensor material and carbides in the structure, $b$ ) carbides and intermediate phases close to the tungsten wire.

Among other observations it was found that $\mathrm{C} 1$ has a graphite free zone around the tube. There is a minor tendency to form cracks at the tube growing into the iron. However, these are very small. In sample $\mathrm{C} 2$ the tube is in the middle of the defect. However, it seems like there are no cracks, and consequently no iron in the tube, as was found on sample B2. On sample C4 a deviating phase has formed. Enormous crack around that makes it look like different solidification structures. One important conclusion is that different problems may appear in SGI compared to LGI. This means that it can be necessary to have different solutions for the different cast iron materials. It can also be concluded that the question about insulator material or not is of paramount importance as that has a consequence on what kind of problems that form. Insulating material will not create a metallurgical continuous interface but will at the same time deteriorate the microstructure to a lesser extent. A metallurgical interface is easier formed between the iron and a metallurgical sensor wire, but that will also increase the risk for carbides and other anomalies in the microstructure. 
In the present experiment no preparation of the sensor/insulator material was done. In a next step coated or in other ways prepared sensor wires/insulators will be tested. Also the process to produce the sensor package can be used to enhance the integration of the sensor into the iron. This could include production under vacuum etc.

\section{Summary}

The main purpose of this part of the project was to perform initial investigations of the interaction between cast iron and different materials acting as sensors or insulators in order to generate empirical data and knowledge. It can be concluded that many of the metallurgical challenges earlier identified have been confirmed by this investigation.

- Large problems with defects. However, as there were no similar defects in the lamellar iron, it is not possible to say that the defects in the ductile iron are due to the sensor/insulator materials or due to other reasons.

- Degenerated structures were found in some cases.

- Air gaps between sensor/insulator material and the iron was in some cases identified.

- Carbides and other intermediate phases have been found.

However, some of these problems can be solved by intermediate layers or additional processes. There are different problems and by that a need for different sensor solutions depending on the cast iron material. The question about insulator material or not will also be important for the final result and quality of the integrated sensor. The starting point for this work was that the sensors or insulator materials cause quality problems in the castings. However, also the opposite was found to be a possible problem; how will the sensor functionality be affected by large cavities in the component.

\section{Acknowledgement}

The project "Smart metal castings" is financed by VINNOVA, the Swedish government agency working under the Ministry of Enterprise and Innovation. Partners within the project are apart from Swerea SWECAST and RISE Acreo AB also Husqvarna AB and SKF Mekan AB.

\section{References}

[1] R. Carlsson, L. Elmquist, C. Johansson, Cast metal with intelligence - from passive to intelligent cast components, VIII ECCOMAS Thematic Conference on Smart Structures and Materials, SMART 2017.

[2] International Conference on System-Integrated Intelligence, www.sysint-conference.org, March 72017.

[3] European Technology Platform on Smart Systems Integration, www.smart-systemsintegration.org, March 72017.

[4] D. Lehmhus, W. Lang, S. Bosse, Mateiral-Integrated Sensing and Intelligent Systems. A review, EPoSS Annual Forum, Cork, September 26, 2013.

[5] Fraunhofer IFAM, www.ifam.fraunhofer.de, March 72017.

[6] D. De Baere et al, Effective Structural Health Monitoring with Additive Manufacturing, EWSHM $-7^{\text {th }}$ European Workshop on Structural Health Monitoring, Jul 2014, Nantes, France, 2014.

[7] J. Kobliska et al, Rapid Fabrication of Smart Tooling with Embedded Sensors by Casting in Molds made by Three Dimensional Printing, Department of Mechanical Engineering, University of Wisconsin-Madison, USA, Madison, 2005.

[8] US Patent, Apparatus and method for embedding optical fibers in metal, US 5283852 A, 1994. 